\title{
Evaluación del rendimiento de nueve genotipos de quinua (Chenopodium quinoa Willd.) bajo diferentes disponibilidades hídricas en ambiente mediterráneo
}

\author{
Grain yield of nine quinoa genotypes (Chenopodium quinoa Willd.) \\ grown in Mediterranean environments differing in water availability
}

\author{
Marco Garrido ${ }^{1}$ Paola Silva ${ }^{1 *}$, Herman Silva ${ }^{1}$, Rocío Muñoz $^{2}$, Cecilia Baginsky ${ }^{1}, E_{\text {dmundo Acevedo }}{ }^{1}$
}

\begin{abstract}
RESUMEN
La quinua es un pseudocereal andino destacado como un cultivo que tolera la sequía; sin embargo, existen pocos estudios que asocien la intensidad del estrés hídrico con la reducción del rendimiento. El objetivo de este trabajo fue evaluar el rendimiento de nueve genotipos de quinua de diferentes procedencias en cuatro condiciones de disponibilidad hídrica y analizar su interacción con el ambiente. Los nueve genotipos de quinua se sometieron a dos disponibilidades hídricas (riego y secano) durante dos temporadas en la zona central de Chile, generándose cuatro ambientes diferenciados principalmente por el monto de agua recibida y su distribución durante la temporada, E1 (487,8 mm), E2 $(245,9 \mathrm{~mm})$, E3 $(660 \mathrm{~mm})$, E4 $(567,1 \mathrm{~mm})$. El rendimiento de los genotipos de quinua se redujo a menos del 50\% cuando se regó al $44 \%$ y $80 \%$ de agua de ETo. El análisis de varianza indicó interacciones significativas entre el genotipo y el ambiente para el rendimiento, índice de cosecha y granos por metro cuadrado. El análisis de componentes principales indicó una asociación fuerte y significativa del rendimiento con el índice de cosecha y los granos por metro cuadrado, una escasa variabilidad en el rendimiento de los genotipos en ambientes con estrés hídrico severo y una mayor variabilidad cuando no hubo estrés. Se observaron dos megaambientes separados por los años en estudio (E1 y E2 vs E3 y E4), desde el punto de vista de genotipos destacaron BAERII como el de mayor rendimiento y BAER como el más estable en el megaambiente que agrupó a E1 y E2. Mientras que el genotipo 4B-216 fue el de mayor rendimiento y 03-21-079 el más estable en el megaambiente que agrupó a E3 y E4.
\end{abstract}

Palabras clave: interacción GxE, sequía terminal, SREG, estabilidad.

\begin{abstract}
Quinoa is an Andean pseudo cereal reputed to be drought resistant. Few studies, however, have related drought intensity to grain yield. The objective of this work was to evaluate the grain yield and its interaction with the environment of nine quinoa genotypes of different origin under four watering environments. The genotypes were exposed to two watering regimes (dryland and irrigated) over two seasons in Central Chile. The watering regimes were characterized by the amount and water distribution during the growing season. E1 $(487.8 \mathrm{~mm}), E 2(245.9 \mathrm{~mm}), E 3(660 \mathrm{~mm}), E 4(567.1 \mathrm{~mm})$. The yield of the genotypes was reduced to less than $50 \%$ when irrigated at 44 and $80 \%$ ETo. There were significant interactions between genotype and environment for grain yield, harvest index, and grains $m$-2. A PCA showed a strong and significant association among yield, harvest index and grains $m-2$, a low variability among genotypes when stressed and a much higher variability when the stress was not present. Two mega-environments were separated by year (E1 and E2 vs E3 and E4). The genotype BAER II had the highest yield and Baer was the most stable in E1 and E2. The genotype 4B-216 had the highest yield and 03-21-079 was the most stable in the megaenvironment grouping E3 and E4.
\end{abstract}

Key words: GxE interaction, terminal drought, SREG, stability

\section{Introducción}

La agricultura requiere aumentar su producción de acuerdo con el crecimiento de la población mundial, que ocurre al mismo tiempo que hay una disponibilidad de agua cada vez menor (Howell,
2001). En zonas con restricción hídrica se deben seleccionar especies o genotipos capaces de producir bajo condiciones de estrés. La quinua (Chenopodium quinoa Willd.) es un pseudocereal cultivado en la zona andina de Perú y Bolivia por más de 7.000 años (Pearsall, 1992) y aparece como un cultivo de

1 Laboratorio de Relación Suelo-Agua-Planta, Facultad de Ciencias Agronómicas, Universidad de Chile. Casilla 1004. Santiago, Chile.

2 Universidad Arturo Prat, Iquique, Chile.

* Autor por correspondencia: psilva@uchile.cl

Fecha de Recepción: 21 Febrero, 2013.

Fecha de Aceptación: 08 Marzo, 2013. 
gran interés para producir en ambientes hídricamente restrictivos (Vacher, 1998; García et al., 2003). En esta especie se han descrito mecanismos de resistencia a la sequía (Jensen et al., 2000; Jacobsen et al., 2003) generalmente asociados a una regulación de la conductancia estomática como punto de control del flujo neto de agua y $\mathrm{CO}_{2}$ (Lawlor, 2001). Pese a esto, muy a menudo se observan reducciones importantes en el rendimiento por estrés hídrico, particularmente cuando el estrés hídrico ocurre en prefloración, floración y grano lechoso (Geerts et al., 2008). La zona de origen de quinua es compleja y variada en factores ambientales que afectan tanto la calidad como el rendimiento de los cultivos presentes en ellos (Aguilar y Jacobsen, 2003), y como consecuencia se observa una alta variabilidad genética (Fuentes et al., 2009) que puede ser utilizada para seleccionar y mejorar genotipos que se adapten a condiciones desfavorables de suelo y clima (García et al., 2003).

Una de las principales limitantes en la selección de variedades, es la alta interacción genotipo $\mathrm{x}$ ambiente (GxE) del rendimiento (Rodríguez et al., 2002). Zobel et al. (1988) compararon técnicas estadísticas incluyendo ANDEVA, análisis de componentes principales y regresiones lineales, demostrando que no siempre son útiles en el análisis de ambientes múltiples. La mayoría de estas técnicas son capaces de identificar la interacción, pero normalmente no permiten realizar un análisis de ella. Una herramienta útil al analizar información obtenida de ensayos multiambiente son los modelos de regresión por sitio (SREG) (Yan et al., 2000). En este modelo el efecto del genotipo y el de GxE constituyen las fuentes de variación. Estos efectos son gráficamente visualizados en un biplot GGE. Este modelo fue propuesto para explorar la respuesta del genotipo a ambientes específicos, ya que agrupa ambientes donde los genotipos tienen una conducta similar (Yan et al., 2000). El objetivo del presente trabajo fue evaluar el rendimiento de nueve genotipos de quinua de diferentes procedencias en cuatro condiciones de disponibilidad hídrica y analizar su interacción con el ambiente.

\section{Materiales y Método}

\section{Condición edafoclimática}

El experimento se realizó en la Estación Experimental Antumapu de la Universidad de Chile (3340'S y 70³8' O, 604 m.s.n.m.). El clima es templado mesotermal estenotérmico mediterráneo semiárido con $330 \mathrm{~mm}$ de precipitación media anual, concentrada $(80 \%)$ entre mayo y agosto, la temperatura media varía entre una máxima de $28,7{ }^{\circ} \mathrm{C}$ en enero y una mínima media de $3,4{ }^{\circ} \mathrm{C}$ en julio (Santibáñez y Uribe, 1990). El suelo es un Mollisol de textura franco arcillo arenosa perteneciente a Serie Santiago, de buen drenaje, profundidad media $(60 \mathrm{~cm}), 2 \%$ de materia orgánica y $\mathrm{pH} 8$.

\section{Diseño experimental y manejo del cultivo}

El experimento fue llevado a cabo durante las temporadas 1998-1999 y 1999-2000. Se evaluaron nueve genotipos de quinua (Cuadro 1) sembrados de forma manual en parcelas de cuatro hileras separadas a $40 \mathrm{~cm}$ y de $10 \mathrm{~m}$ de largo. El diseño fue de bloques completos al azar con dos repeticiones.

La dosis de siembra fue de $10 \mathrm{~kg} \mathrm{ha}^{-1}$. La fecha de siembra fue el 24 de julio de 1998 y 6 de agosto de 1999. La fertilización fue de 100 unidades de nitrógeno (urea) y 80 unidades de $\mathrm{P}_{2} \mathrm{O}_{5}$ (superfosfato triple) aplicados a la siembra. Las malezas fueron controladas de forma manual, no se presentaron ni plagas ni enfermedades en el cultivo. El mismo manejo fue repetido ambos años.

El ensayo se ubicó en una línea de aspersión similar a la descrita por Hanks et al. (1976), lo que permitió obtener diferentes cargas de agua de acuerdo con la cercanía del genotipo a la línea de aspersión. Los genotipos próximos a la línea de aspersión recibieron $100 \%$ del agua utilizada por el cultivo (bien regado), y los genotipos distales no lograron suplir su requerimiento hídrico (déficit hídrico). Cada lado de la línea de aspersión constituyó una repetición. Cada evento de riego ocurrió cuando el tratamiento bien regado consumió el 50\% de la humedad aprovechable del suelo. El contenido de

Cuadro 1. Genotipos de quinua y su procedencia.

\begin{tabular}{lll}
\hline Nombre del genotipo & Ecotipo & País de procedencia \\
\hline I-109 & Altiplano & Perú \\
4B-216 & Altiplano & Perú \\
LP-3A2 & Altiplano & Perú \\
$03-21-079$ & Altiplano & Perú \\
$04-02-641$ & Altiplano & Bolivia \\
$03-08-864$ & Altiplano & Perú \\
$03-04-1017$ & Valle & Perú \\
BAER II & Nivel del mar & Chile \\
BAER & Nivel del mar & Chile \\
\hline
\end{tabular}


agua a Capacidad de Campo es de $29 \%$ y Punto de Marchites Permanente de 15\%, además de una profundidad de suelo de $60 \mathrm{~cm}$ y una densidad aparente de $1,3 \mathrm{~g} \mathrm{~cm}^{-3}$. El ensayo se inició a Capacidad de Campo en ambas temporadas.

\section{Variables medidas}

Se midió la biomasa, el rendimiento y sus componentes; peso seco de 1.000 granos (PMG) y granos por metro cuadrado (GMC) y se calculó el índice de cosecha (IC). Para ello $1 \mathrm{~m}^{2}$ de plantas por unidad experimental fueron cosechados manualmente y secados en estufa a $60{ }^{\circ} \mathrm{C}$ hasta peso constante. Los valores fueron expresados a $0 \%$ de humedad. El índice de cosecha fue determinado para cada muestra como el cociente entre el rendimiento en grano y la biomasa aérea. El peso de 1.000 granos fue estimado a partir de 50 semillas obtenidas al azar de cada muestra cosechada.

La temperatura máxima, mínima, evaporación de bandeja y precipitación, fue obtenida de la estación meteorológica del Instituto de Investigaciones Agropecuarias (INIA), Centro Regional de Investigación La Platina, ubicada a 700 m del ensayo.

\section{Análisis estadístico}

Se realizó análisis combinado de varianza para evaluar el rendimiento y componentes del rendimiento de los genotipos y su interacción en cada ambiente. Se realizó análisis de componentes principales con gráficos Biplot, de manera de conocer la asociación entre el rendimiento y sus componentes e identificar grupos de genotipos. Para explorar la variabilidad del GENOTIPO + GENOTIPO x AMBIENTE se utilizó el modelo lineal-bilineal SREG (Crossa et al., 2002). Los análisis fueron realizados con el software estadístico InfoGen versión 2012 (Balzarini y Di Rienzo, 2012).

\section{Resultados y Discusión}

\section{Condición ambiental}

La temperatura media diaria del aire fue similar entre estaciones, al contrario de la precipitación, la cual fue contrastante (Figura 1). La temperatura media del aire y precipitación acumulada fueron $15,1^{\circ} \mathrm{C}$ y $21,6 \mathrm{~mm}$ para la temporada $1998-1999$, y $15,6^{\circ} \mathrm{C}$ y $268,9 \mathrm{~mm}$ para la temporada $1999-2000$.

El total de agua recibida (precipitación + riego) en las parcelas bien regadas y con déficit hídrico en la temporada 1998-1999 fue de 487,8 y 245,9 mm supliendo un $86 \%$ y un $44 \%$ de la demanda evapotranspirativa, respectivamente. Durante la temporada 1999-2000 los montos fueron de $660 \mathrm{~mm}$ para las parcelas bien regadas y $567,1 \mathrm{~mm}$ para las parcelas con déficit hídrico, supliendo un $93 \%$ y un $80 \%$ de la demanda evapotranspirativa del cultivo.

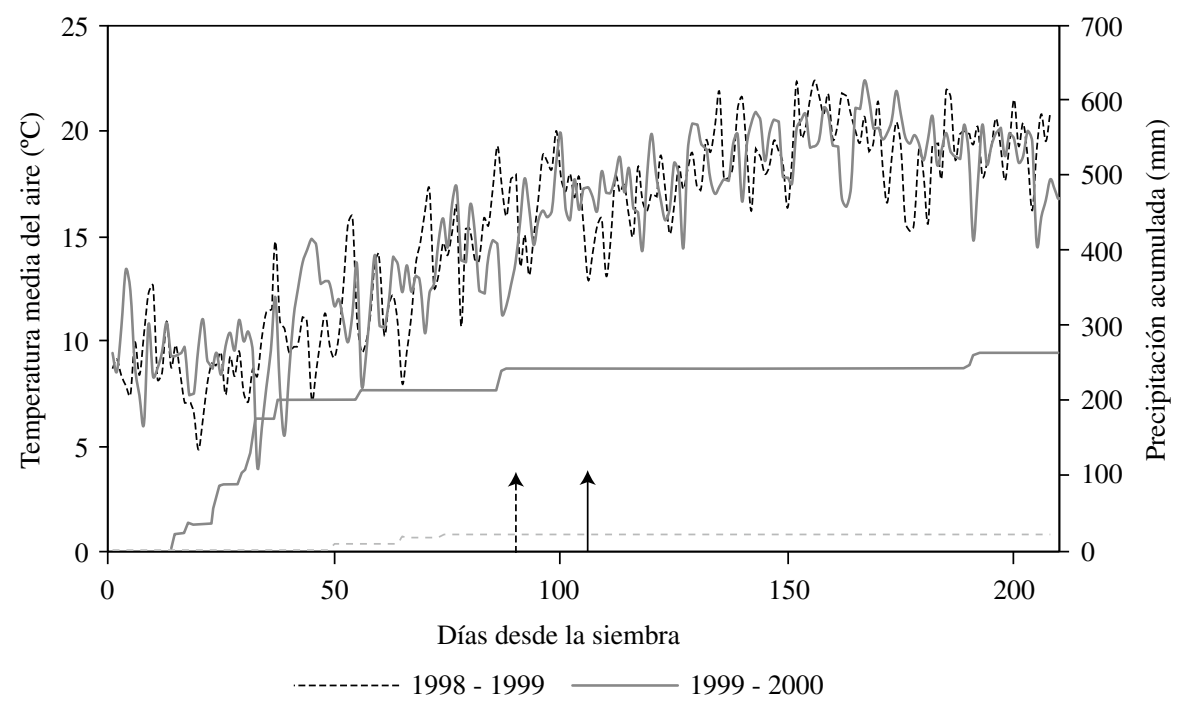

Figura 1. Temperatura media diaria del aire y precipitación acumulada durante el periodo de crecimiento del cultivo de quinua en las temporadas 1998-1999 y 1999-2000. Flecha continua: Floración 1998-1999, Flecha discontinua: Floración 1999-2000. 
La sequía experimentada en la primera temporada expuso a los genotipos bajo déficit hídrico a un estrés severo inmediatamente después de floración (Figura 2a), a diferencia de la segunda temporada (Figura 2b), la cual experimentó, incluso, periodos prolongados de anegamiento en etapas tempranas de desarrollo del cultivo (hasta 40 días después de la siembra).

\section{Rendimiento y componentes del rendimiento}

Los ambientes se ordenaron de mayor a menor rendimiento promedio en la siguiente secuencia E3; E1; E4 y E2 con 2.244, 1.900, 823 y $620 \mathrm{~kg}$ $\mathrm{ha}^{-1}$, respectivamente (Cuadro 2). El rendimiento promedio de los genotipos de quinua fluctuó entre 754 y $2.032 \mathrm{~kg} \mathrm{ha}^{-1}$. Los genotipos de quinua en E2 y E4 solo suplieron el $44 \%$ y $80 \%$ del agua de ETo, respectivamente, y muy pocos genotipos lograron alcanzar el 50\% de su rendimiento potencial. El bajo rendimiento observado en E4 y E2 se puede explicar por el estrés hídrico ocurrido en las etapas de prefloración, floración y grano lechoso, las cuales se consideran como las más sensibles a estrés hídrico en quinua (Geerts et al., 2008). Otros autores han señalado una alta sensibilidad en quinua en etapas de desarrollo similares para maíz (Yilmaz et al., 2006) y trigo (Zhang et al., 2006). La fuerte reducción del rendimiento contradice la aseveración de Vacher (1998), quien consideró al cultivo tolerante al estrés hídrico debido a que logró un rendimiento promedio de $2.345 \mathrm{~kg} \mathrm{ha}^{-1}$ cuando sometió dos genotipos de quinua a una relación entre
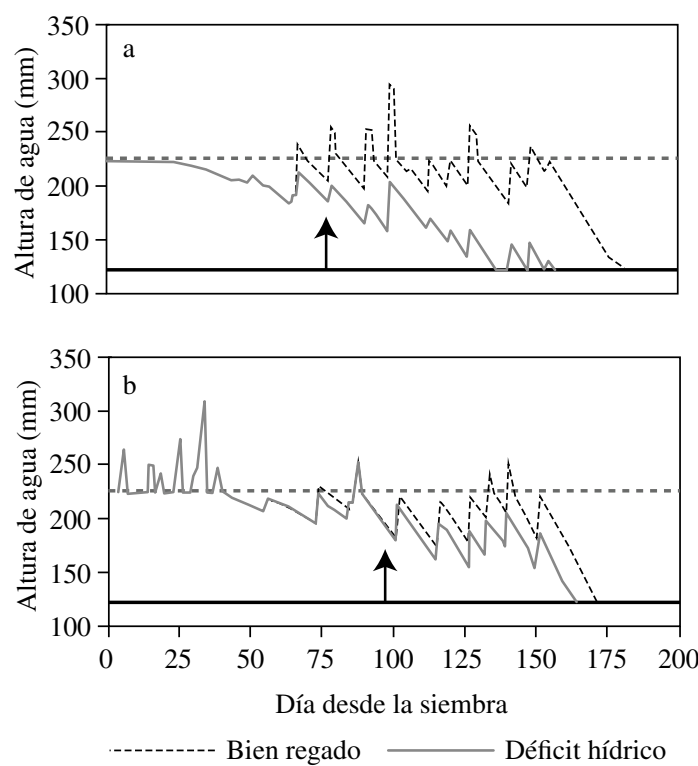

Figura 2. Balance del agua en el suelo para la temporada 1998-1999 (a) y temporada 1999-2000 (b). La línea segmentada paralela al eje x corresponde a capacidad de campo (226 mm) y línea continua a punto de marchitez permanente $(122 \mathrm{~mm})$. La flecha indica el día promedio de floración de los genotipos evaluados.

precipitación y evapotranspiración de referencia (pp/ETo) de 0,32 durante los últimos 52 días del ciclo, entre floración y llenado de granos. Sin embargo, este trabajo careció de un tratamiento sin estrés que permitiera conocer la reducción del rendimiento potencial del cultivo. La misma deficiencia se observa en el trabajo de González et al. (2011) que evaluó

Cuadro 2. Rendimiento promedio de los genotipos de quinua en cada ambiente.

\begin{tabular}{lcccrr}
\hline $\begin{array}{c}\text { Genotipo } \\
-\end{array}$ & $\begin{array}{c}\text { E1 } \\
\left(\mathrm{kg} \mathrm{ha}^{-1}\right)\end{array}$ & $\begin{array}{c}\text { E2 } \\
\left(\mathrm{kg} \mathrm{ha}^{-1}\right)\end{array}$ & $\begin{array}{c}\text { E3 } \\
\left(\mathrm{kg} \mathrm{ha}^{-1}\right)\end{array}$ & $\begin{array}{c}\text { E4 } \\
\left(\mathrm{kg} \mathrm{ha}^{-1}\right)\end{array}$ & $\begin{array}{r}\text { Promedio } \\
\left(\mathrm{kg} \mathrm{ha}^{-1}\right)\end{array}$ \\
\hline I-109 & 1.049 & 264 & 2.432 & 813 & 1.140 \\
4B-216 & 1.242 & 723 & 4.168 & 909 & 1.760 \\
LP-3A2 & 2.391 & 227 & 3.107 & 1.090 & 1.704 \\
03-21-079 & 1.134 & 491 & 2.813 & 1.294 & 1.433 \\
04-02-641 & 874 & 403 & 1.805 & 690 & 943 \\
03-08-864 & 2.929 & 317 & 1.268 & 401 & 1.228 \\
03-04-1017 & 663 & 275 & 1.548 & 531 & 754 \\
BAER II & 3.983 & 1.663 & 1.588 & 796 & 2.032 \\
BAER & 2.837 & 1.218 & 1.471 & 823 & 1.578 \\
Promedio & 1.900 & 620 & 2.244 & 91 & 1.397 \\
E.E. & 388 & 167 & 322 & & 138 \\
\hline
\end{tabular}

E1 = Temporada 1998-1999 bien regado; E2 = Temporada 1998-1999 con déficit hídrico; E3 = Temporada 1999-2000 bien regado; E4 = Temporada 1999-2000 con déficit hídrico; E.E. = Error estándar. 
10 cultivares de quinua bajo secano ( $\mathrm{pp}$ ETo $=0,38$ ), obteniendo rendimientos que variaron entre $376 \mathrm{y}$ $3.855 \mathrm{~kg} \mathrm{ha}^{-1}$, destacando la variabilidad genotípica del rendimiento bajo condiciones de estrés, pero sin señalar su tolerancia a este estrés. En este trabajo se señala que los genotipos con mayor rendimiento bajo estrés tienen una mayor conductancia estomática y mayor transpiración, lo que concuerda con lo señalado por Blum (2009), para otros cultivos. Adicionalmente, se ha informado rendimientos en quinua de hasta $7.700 \mathrm{~kg} \mathrm{ha}^{-1}$ sin estrés y 4.900 $\mathrm{kg} \mathrm{ha}^{-1}$ con reducción del riego del $50 \%$ (Martínez et al., 2009).

\section{Interacción genotipo por ambiente}

En el Cuadro 3 se observa el peso relativo de ambiente, genotipo y de la GxE para distintas variables productivas. La variación en rendimiento está principalmente explicada por interacción $\mathrm{GxE}$, siendo su efecto tres veces mayor que el de genotipo, ocurriendo algo similar con GMC y el IC. Por otra parte, la biomasa y el PMG no tuvieron interacción GxE ni efecto de genotipo ni ambiente. La presencia de la interacción GxE para rendimiento pone en evidencia el problema de seleccionar genotipos bajo alguna condición específica ignorando la interacción GxE (Bertero et al., 2004).

Dada la interacción GxE sobre el rendimiento, IC y GMC, se realizó un análisis de componentes principales para analizar la tendencia de los datos y la asociación entre ellos (Figura 3).

Los componentes CP1 y CP2 explicaron el 87,8\% de la variación total observada. El rendimiento se asoció positiva y significativamente con IC y GMC. A su vez GMC se asoció con fuerza con la biomasa producida. A nivel de la CP1, la condición hídrica favorable se separó de la desfavorable siendo el rendimiento, seguido por el IC y GMC las variables de mayor peso relativo en CP1. La CP2 separa los ambientes según año, siendo las variables biomasa y PMG las responsables de la variación observada en este componente.

Cuando los genotipos se encontraban en un buen ambiente, se observó una mayor variabilidad del rendimiento entre genotipos, a diferencia de los ambientes restrictivos, donde la variación fue menor, resultado que discrepa con lo observado por Pulvento et al. (2010) quienes evaluaron los genotipos de origen chileno Regalona BAER y KVLQ520Y bajo condiciones de secano en clima mediterráneo al sur de Italia durante dos temporadas. Cuando la precipitación fue mayor y más homogénea los genotipos no se diferenciaron en términos de rendimiento $\left(3.280\right.$ y $3.420 \mathrm{~kg} \mathrm{ha}^{-1}$ para KVLQ520Y y Regalona BAER, respectivamente), mientras que en la temporada siguiente, cuando las precipitaciones cesaron temprano y por tanto se dieron condiciones de estrés, los genotipos tuvieron marcadas diferencias (1.900 y $3.000 \mathrm{~kg} \mathrm{ha}^{-1}$ para KVLQ520Y y Regalona BAER, respectivamente).

Dada la alta variación observada se procedió a realizar una selección de genotipos por sitio. El análisis combinado de varianza (Cuadro 3) muestra que el efecto del ambiente sobre el rendimiento fue de $45,0 \%$ de la suma de cuadrados, mientras que el del genotipo fue de $13,8 \%$. La magnitud de la variación atribuida a $\mathrm{GxE}$ fue de $41,2 \%$, tres veces superior a la de G, sugiriendo la posibilidad de agrupar ambientes de acuerdo con la interacción GxE (Yan et al., 2000) mediante un modelo SREG (Crossa et al., 2002).

El modelo SREG explicó el 93,2\% de la variación de la interacción GxE (Figura 4). Los genotipos que conformaron el polígono envolvente fueron BAER II, 03-04-1017, 4B-216 y 03-08-864. Estos genotipos vértices son los que más aportan a la interacción, es decir, los de más alto y más bajo

Cuadro 3. Porcentaje relativo a la suma de cuadrados totales del análisis combinado de varianza para biomasa, rendimiento y componentes de rendimiento.

\begin{tabular}{lccccc}
\hline F.V. & $\begin{array}{c}\text { Biomasa } \\
\left(\mathrm{kg} \mathrm{ha}^{-1}\right)\end{array}$ & $\begin{array}{c}\text { Rendimiento } \\
\left(\mathrm{kg} \mathrm{ha}^{-1}\right)\end{array}$ & $\begin{array}{c}\text { IC } \\
-\end{array}$ & $\begin{array}{c}\text { PMG } \\
(\mathrm{g})\end{array}$ & $\begin{array}{c}\text { GMC } \\
-\end{array}$ \\
\hline Ambiente & $81,7^{\text {n.s. }}$ & $45,0^{* * *}$ & $49,7^{* * * *}$ & $57,1^{\text {n.s. }}$ & $44,9^{\text {n.s. }}$ \\
Genotipo & $4,8^{\text {n.s. }}$ & $13,8^{* * *}$ & $0,5^{* * *}$ & $10,5^{\text {n.s. }}$ & $14,3^{* * *}$ \\
GxE & $13,5^{\text {n.s }}$ & $41,2^{* * *}$ & $39,8^{* * *}$ & $32,4^{\text {n.s. }}$ & $40,8^{* * *}$ \\
\hline
\end{tabular}

F.V. = Fuente de variación; $\mathrm{IC}=$ Índice de cosecha; PMG = Peso seco de 1.000 granos; $\mathrm{GMC}=$ Granos por metro cuadrado de suelo; $*=(\mathrm{p} \leq 0,05) ; * *=(\mathrm{p} \leq 0,01) ; * * *=(\mathrm{p} \leq 0,001) ;$ n.s. $=$ No significativo. 


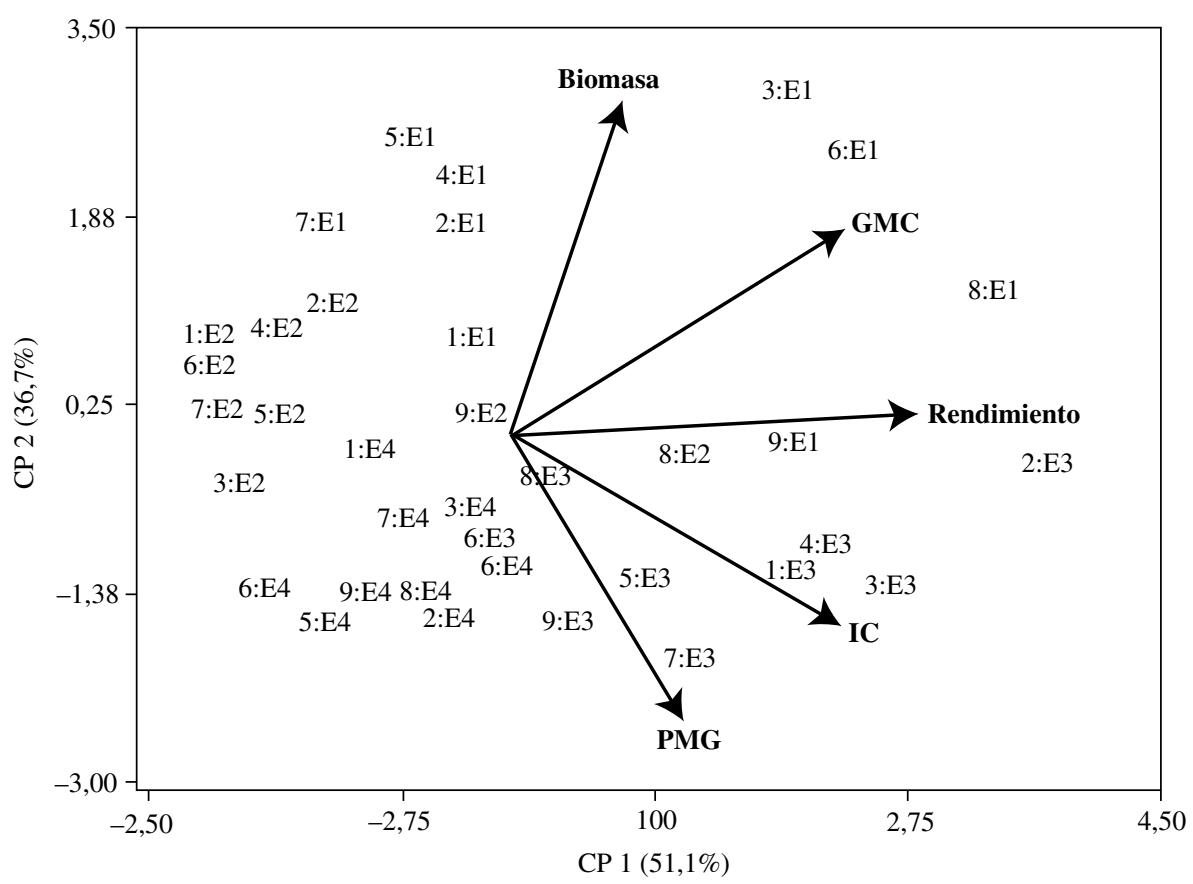

Figura 3. Biplot de los primeros dos componentes principales para biomasa, rendimiento, índice de cosecha (IC), peso seco de 1.000 granos (PMG), granos por metro cuadrado (GMC) para nueve genotipos de quinua evaluados en cuatro ambientes. El primer número corresponde al genotipo y el segundo al ambiente.

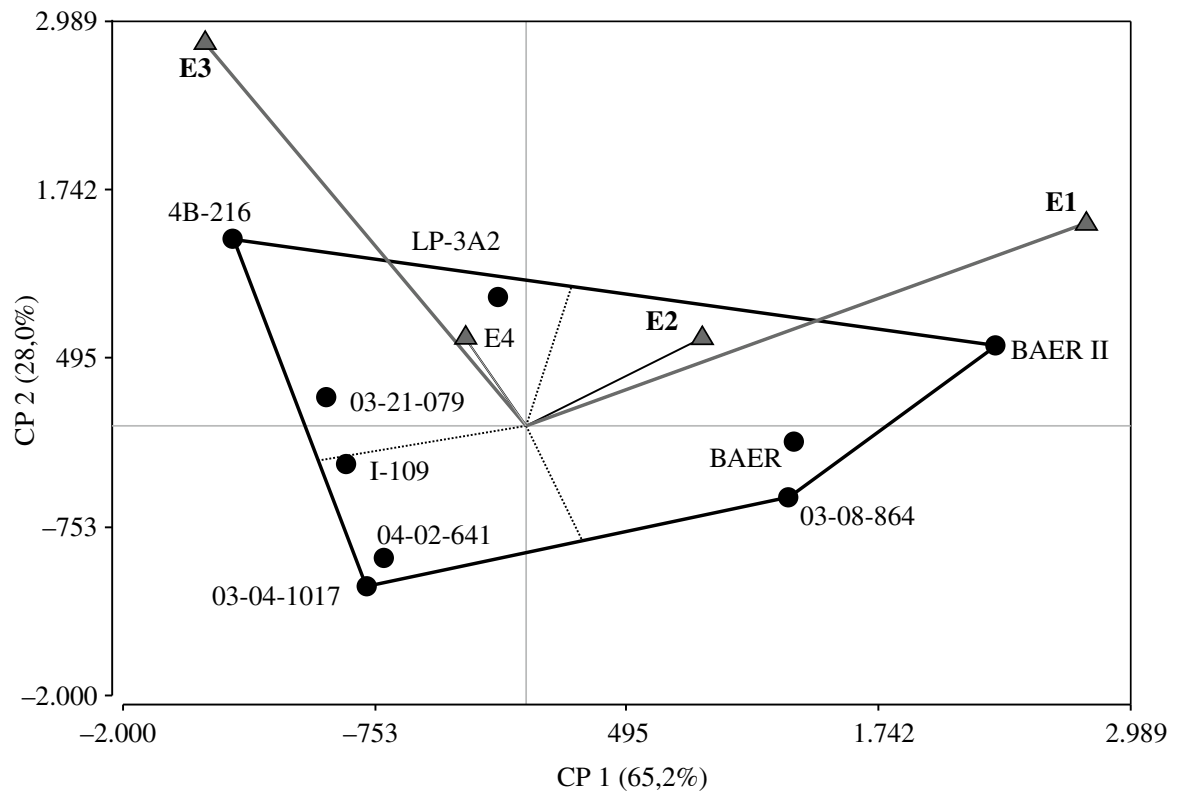

Figura 4. Biplot GGE para rendimiento de grano de nueve genotipos de quinua evaluados en cuatro ambientes. 
rendimiento. Las líneas perpendiculares a cada uno de los lados del polígono y que pasan por el origen del biplot definen ambientes hipotéticos (o megaambientes) donde los genotipos contenidos tienen desempeños similares (Yan y Rajcan, 2002). Se definieron dos megaambientes, el que se compone por E1 y E2 correspondientes a la temporada 1998-1999 caracterizado por una baja pluviometría al inicio de la temporada, donde BAER II, BAER y 03-08-864 son los genotipos más adaptados. El segundo megaambiente está compuesto por E3 y E4, correspondiente a la temporada 2009-2010, con una alta pluviometría al inicio de la temporada, donde 4B-216, 03-21-079 y LP-3A2 fueron los genotipos mejor adaptados. Los genotipos I-109, 04-02-641 y 03-04-1017 son los de menor rendimiento en todos los ambientes. Los genotipos más cercanos al valor cero del CP2 fueron los más estables, es decir, fueron los que tuvieron menor respuesta ante cambios en el ambiente. En función de lo anterior, los genotipos más interesantes son los que tienen un valor alto en el CP1 y bajo en el CP2, ya que tendrían rendimientos altos y estables, en este sentido en el megaambiente formado por E2 y E1, BAER II es el de mayor rendimiento, seguido por el genotipo estable BAER. En el megaambiente compuesto por E3 y E4 el genotipo de mayor rendimiento fue 4B-216 seguido por el genotipo estable 03-21-079.

Cabe destacar la tendencia de los genotipos según su origen. En el megaambiente E1 E2 hubo un mejor desempeño de los genotipos chilenos, mientras que en el megaambiente E3 E4 los de origen peruano y boliviano tuvieron un mejor desempeño.

\section{Conclusiones}

El rendimiento de quinua es fuertemente afectado por la sequía terminal, su efecto cambia con el genotipo y el ambiente, destacándose la importancia de seleccionar genotipos de mayor rendimiento bajo condiciones de estrés hídrico considerando GxE. En ausencia de estrés los genotipos de quinua evaluados mostraron las mayores diferencias de rendimiento, no obstante en condiciones de estrés el rendimiento tendió a homogeneizarse, por lo que al parecer, los genotipos probados no poseen caracteres particulares que les permitan destacarse ante condición de estrés hídrico severo.

\section{Literatura Citada}

Aguilar, P.C.; Jacobsen, S.E.

2003 Cultivation of quinoa on the Peruvian Altiplano. Food Rev. Int., 19: 31-41.

Balzarini, M.G.; and Rienzo, J.A. Di.

2012 InfoGen versión 2012. FCA, Universidad Nacional de Córdoba, Argentina. URL http://www.info-gen.com.ar

Bertero, H.; De la Vega, A.; Correa, G.; Jacobsen, S.; Mujica, A. 2004 Genotype and genotype-by-environment interaction effect for grain yield and grain size of quinoa (Chenopodium quinoa Willd.) as revealed by pattern analysis of internal multi-environment trials. Field Crops Res., 89: 299-318.

Blum, A.

2009 Effective use of water (EUW) and not water use efficiency (WUE) is the target of crop yield improvement under drought stress. Field Crops Res., 112: 119-123.

Crossa, J.; Cornelius, P.L.; Yan, W.

2002 Biplots of linear-bilinear models for studying crossover genotype x environment interaction. Crop Sci., 42 : 619-633.

Fuentes, F.; Maughan, P.; Jellen, R.

2009 Diversidad genética y recursos genéticos para el mejoramiento de la quinoa (Chenopodium quinoa Willd.). Rev. Geogr. Valpso., 42: 20-33.

García, M.; Raes, D.; Jacobsen, S.

2003 Evapotranspiration analysis and irrigation requirements of quinoa (Chenopodium quinoa) in the Bolivian highlands. Agric. Water Manage, 60: 119-134.
Geerts, S.; Raes, D.; García, M.; Vacher, J.; Mamani, R.; Mendoza, J.; Huanca, R.; Morales, B.; Miranda, R.; Cusicanqui, J.; Taboada, C.

2008 Introducing deficit irrigation to stabilize yields of quinoa_(Chenopodium quinoa Willd.). Eur. J. Agron., 28: 427-436.

González, J.A.; Bruno, M; Valoy, M; Prado, F.E.

2011 Genotypic variation of gas exchange parameters and leaf stable carbon and nitrogen isotopes in ten quinoa cultivars grown under drought. J. Agron. Crop Sci., 197: 81-93.

Hanks, R.J.; Keller, V.; Rasmussen, A.; Wilson, G. 1976 Line source sprinkler for continuous variable irrigationcrop production studies. Soil Sci. Soc. Am. J. 40: 426-429.

Howell, T. 2001: Enhancing water use efficiency in irrigated agriculture. Agron. J., 93: 281-289.

Jacobsen, S.; Mujica, A.; Jensen, C. 2003 The resistance of quinoa (Chenopodium quinoa Willd.) to adverse abiotic factors. Food Rev. Int., 19: 99-109.

Jensen, C.; Jacobsen, S.; Anderson, M.; Nuñez, N.; Anderson, S.; Rasmussen, L.; Mogensen, V.

2000 Leaf gas exchange and water relation characteristics of field quinoa (Chenopodium quinoa Willd.) during soil drying. Eur. J. Agron., 13: 11-25.

Lawlor, D. W.

2001 Photosynthesis, $3^{\text {rd }}$ edn. BIOS Scientific Publishers Ltd, New York, USA. 386 p. 
Martínez, E. A.; Veas, E.; Jorquera, C.; San Martín, R.; Jara, P. 2009 Re-introduction of quínoa into arid Chile: Cultivation of two lowland races under extremely low irrigation. J. Agron. Crop Sci., 195: 1-10.

Pearsall, D.M.

1992 The origins of plant cultivation in South America. In: Cowan, C.W., Watson, P.J. (Eds.), The Origins of Agriculture. Smithsonian Institute Press., Washington, DC, pp. 173-205.

Pulvento, C.; Riccardi, M.; Lavini, A.; D’Andria, R.; Iafelice, A.; Marconi, E.

2010 Field trial evaluation of two Chenopodium quinoa genotypes grown under rain-fed conditions in a typical Mediterranean environment in south Italy. J. Agron. Crop Sci., 196: 407-411.

Rodríguez, J.; Sahagún, J.; Villaseñor, H.; Molina, J.; Martínez, A. 2002 Estabilidad de siete variedades comerciales de trigo (Triticum aestivum L.) de temporal. Rev. Fitotec. Mex., 25: 143-151.

Santibáñez, F.; Uribe, J.

1990 Atlas Agroclimático de Chile. Regiones V y Metropolitana. Universidad de Chile, Facultad de Ciencias Agrarias y Forestales, Santiago, Chile. 65 p.
Vacher, J.

1988 Responses of two main Andean crops, quinoa (Chenopodium quinoa Willd) and papa amarga (Solanum juzepczukii Buk.) to drought on the Bolivian Altiplano: Significance of local adaptation. Agric. Ecosyst. Environ., 68: 99-108.

Yan, W.; Rajcan, I.

2002 Biplot evaluation of test sites and trait relations of soybean in Ontario. Crop Sci., 42: 11-20.

Yan, W.; Huny, L.A.; Sheng, Q.; Szlavnics, Z. 2000 Cultivar evaluation and mega-environment investigation based on the GGE biplot. Crop Sci., 40: 597-605.

Yilmaz, E.; Dagdelen, N.; Sezgin, F.; Bas, S.

2006 Responses of corn (Zea mays L.) to water stress at different growth stages. In: Proceedings of the $1^{\text {st }}$ International Symposium on Land and Water Management for Sustainable Irrigated Agriculture, CD.ROM.

Zhang, B.; Huang, G.; Cheng, Z.; Zhang, Y.

2006 Yield performance of spring wheat improved by regulated deficit irrigation in an arid area. Agric. Water Manage., 79: $28-42$.

Zobel, R.W.; Wright, M.J.; Gauch, H.G.

1988 Statistical analysis of a yield trial. Agron. J. 80: 388-393. 